\title{
Comfort: A Coordinate of User Experience in Interactive Built Environments
}

\author{
Hamed S. Alavi ${ }^{1,2}$, Himanshu Verma ${ }^{1}$, Michael Papinutto ${ }^{1,3}$ and Denis Lalanne ${ }^{1}$ \\ 1 Human-IST Research Center, University of Fribourg, Switzerland \\ 2 Swiss Federal Institute of Technology (EPFL), Lausanne, Switzerland \\ 3 Visual and Social Neuroscience Unit, University of Fribourg, Switzerland \\ \{hamed.alavi, himanshu.verma, michael.papinutto, denis.lalanne\}@unifr.ch
}

\begin{abstract}
Comfort as a technical term in the domain of architecture has been used meticulously to describe, assess, and understand some of the essential qualities of buildings, across four dimensions: visual, thermal, acoustic, and respiratory. This body of knowledge can be drawn upon to shed light on the growing branch of HCI that pursues a shift from "artifact" to "environment" (and from "usability" to "comfort"). We contribute to this conceptual-contextual transition in three consecutive steps: 1) sketch the outline of comfort studies in the scholar field of Architecture and the ones in Human-Computer Interaction, 2) propose a schematic model of comfort that captures its interactive characteristics and, 3) demonstrate an interactive tool, called ComfortBox, that we prototyped to help answer some of the research questions about the perception of comfort in built environments.
\end{abstract}

Keywords: Human-Building Interaction; Comfort; Adaptive Architecture

\section{Introduction}

Interaction design and architecture have a two-decade-long history of mutual influence and dialogue. From one side, interaction design has drawn on architectural concepts and reflections in multitude of endeavours, namely, in the reification of "Ubicomp" vision and the physical embodiment of computing [1], in the amalgamation of function and form [2], in the development of interaction design patterns [3], and in studying the symbiosis of people and artifacts [4]. From the other side, in architecture, numerous attempts strove to understand the integration of digital elements in buildings and urban design [5, 6]. Despite these instances of mutual learning, it has been argued that the depth and breath of concrete collaborations between architects and interaction designers are far from the ideal [7]. The cases where interaction designers or HCI researchers engage in an architectural project are rather rare [8]. Instead, in the current scenarios, technology is typically retrofitted onto a new or existing building, to perform functions that were previously done "less efficiently". The evolution of windows exemplifies this scenario. Window is a remarkably sophisticated architectural element with physical, spatial, and social affordances, which have been the subject of creative design for many years. With the recent ecological and energy concerns, the efficiency of operable windows has been criticized, leading to the situation where in modern buildings the interconnected functions of window are separated and each assigned to one building automation component (e.g. 
automated shading, ventilation, air conditioning). These components (functions) have been occasionally re-envisioned with a user-centered approach, and substituted by interactive technological innovations such as Nest learning thermosta 4 . Comfy Apf ventilation, and context-aware shading and lighting. Questioning this retrofitting model against the ideas of embodiment and the vision of "profound technolgies" in Ubicomp, the notion of Human-Building Interaction (HBI) [9] has proposed an alternative scenario, in which interaction design, and more broadly $\mathrm{HCI}$, have an early interwoven engagement in the design, construction, and evaluation of built environments. This, nevertheless, entails constructing a common grounding about what it means to design user experience with and within built environments, of which comfort is a key coordinate.

Given this background, we present an elaboration on the concept of comfort. The ultimate goal is to create practical knowledge and tools that can support HBI design research. In this paper, we, first, summarize how comfort is studied in architecture, identifying the broad research questions that have been widely investigated, and the ones that are currently the topic of scientific explorations. Second, we propose a model that illustrates the relationships between the components that are found relevant in the existing studies, but also highlights the significance of HBI design and the processes through which comfort is achieved in interaction with the environment. This model has inspired the design of a tool that we prototyped to facilitate the data collection process in comfort studies. The tool, called ComfortBox, is described in the third part of this paper.

In the interest of clarity, we restrain our discussion within the scope that has been well-established in the domain of architecture, covering the four dimensions of thermal, visual, acoustic, and respiratory comfort. In the following text, whenever we use the term "comfort", we refer to only these four dimensions, unless it is explicitly stated otherwise.

\section{Comfort Studies in Architecture}

Gaston Bachelard in his book La Poétique de l'Espace (The Poetics of Space) has compared comfort - in its broadest sense - to the 'lived experience' within architecture as its phenomenological consequence. Similar philosophy has been adopted since the early $21^{\text {st }}$ century to investigate the relationship between human health \& well-being and the physical characteristics of the indoor environment - temperature, air, light and sound [10]. Subsequently, the description of comfort was encapsulated into four respective dimensions (thermal, respiratory, visual, and acoustic), and remained the basis for further design and studies until the present time [11].

The rest of this section aims to chart the outline of the research landscape that investigates these dimensions. This is done through, first and foremost, stating the very broad research questions that have been raised about comfort in the domain of architecture. The answers that we present for each of these questions demonstrate a summary of the influential and iconic works in the respective field, and contribute to our discussion by giving insight into the commonly applied methodologies and the scientific language, which notably differ from those of interaction design research.

\footnotetext{
${ }^{4}$ https://nest.com/uk/thermostat/meet-nest-thermostat/

${ }^{5}$ https://www.comfyapp.com/
} 
Q1. What environmental attributes influence the occupant's comfort?

Thermal comfort has been found to be percieved in relation to the ambient air temperature, humidity, and air-flow. Fanger [12] in his highly influential set of studies used thermal chamber to examine participant's reaction to different thermal conditions. He created a model called the Predicted Mean Vote and Predicted Percent Dissatisfied (PMV/PPD), which led to the standardization of building thermal conditions by the International Organization of Standardization (ISO). Hopkinson [13] has illustrated the different surface and material characteristics that can be used to assess the visual comfort. These characteristic parameters are $a$ ) the amount of light on the surface (i.e. illuminance), $b$ ) the intensity of light reflected by the surfaces (i.e. luminance), $c$ ) temperature of light, and $d$ ) glare. Intensity of noise (in $\mathrm{db}$ ) and its frequency in Herz (Hz) determine the levels of acoustic comfort [11]. Finally, the effects of different constituents of air on human health have been examined, but since some studies have found inconclusive results with regards to different pollutants [14, 15], respiratory comfort is predominantly associated to the concentration of $\mathrm{CO} 2$ (in ppm - Parts Per Million).

Q2. In what delineated ranges of environmental attributes are buildings perceived as comfortable?

Despite the large amount of research conducted to find the ranges of acceptable thermal condition, it has remained the topic of discourse and only two standards (ASHRAE 55 and EN15251) have reached a consensus, recommending the operative temperature range from $21^{\circ} \mathrm{C}$ to $24^{\circ} \mathrm{C}$ in winter and from $22^{\circ} \mathrm{C}$ to $25^{\circ} \mathrm{C}$ in summer [16-18]. Visual comfort is a task-dependent quality; the amount and type of light that is needed depends on the task that the occupant is engaged in. The high precision tasks, for instance, require higher level of illuminance and luminance without glare. CEN [19] provided recommendations regarding glare, colour index and lightning level in an office space (illuminance near 500 lux, luminance in the range $[32,130]$ lux). Different building characteristics such as the location and the type of building have been considered as the factors that define the acceptable acoustic conditions. These characteristics are quantified via the levels of the Noise Ratio (NR), which varies for different buildings. For example, the recommended Noise Ratio level for an office is defined as 35 [20]. Finally, Multitude of research on air-quality levels, and its subsequent standardization for habitable buildings by ANS17] ASHRAE [21], has specified the recommended concentrations of $\mathrm{CO} 2$ levels to be below $1000 \mathrm{ppm}$ for a higher respiratory comfort.

Q3. How do the individual and cultural characteristics influence these ranges?

Surprisingly, the study of comfort as a subjective phenomenon has been delayed until the $21^{\text {st }}$ century. Brager and de Dear in their 2007 book, Buildings, Culture and Environment: Informing Local and Global Practices, state that "comfort is not just an outcome of the physical environment but it is our very attitudes about comfort both on an individual and cultural scale" [22]. Two recent studies by Chappels and Shafaghat uncover the intraindividual and inter-individual variability, indicating differences of comfort sensations depending on culture, experience, age, and education [23, 24].

\footnotetext{
${ }^{6}$ American Society of Heating, Refrigerating and Air-Conditioning Engineers

${ }^{7}$ American National Standards Institute
} 


\section{Q4. How can a built environment be assessed in terms of comfort?}

Post-Occupancy Evaluation (POE) is a commonly used method to assess buildings in terms of their inhabitants' comfort and living experiences, after they have been occupied [25]. Questionnaires have often been used to evaluate occupants' perceived comfort along the aforementioned dimensions, and consequently identify different kinds of architectural or constructional flaws [26, 27]. In a lab setting, other methods such as the analysis of physiological responses have been increasingly incorporated to speculate the level of experienced comfort. For instance, Yao and his colleagues demonstrated that different physiological indicators such as skin temperature, heart rate, and electroencephalography (EEG) can be used as reliable predictors of perceived thermal comfort [28, 29].

\section{Comfort Studies in HCI}

Driven by the move towards sustainable habitation, studies focusing on occupants' comfort have been a relatively recent trend in HCI and Ubicomp. Thermal comfort, amongst the different dimensions, has been the popular subject of investigation because of the $a$ ) perceived lack of control and engagement within tightly controlled, automated buildings; and $b$ ) enhanced measures to conserve energy through contextual optimization of HVAC ${ }^{8}$ systems. These aspects have significantly pronounced the role of HCI in Sustainability [30], and driven the research agenda towards the design of many user-oriented interactive artifacts, where thermal comfort and awareness about energy utilization are (strongly) coupled and contextualized. Milenkovic et al. [31], for example, have demonstrated an energy-awareness system called POEM (Personal Office Energy Monitor) that also enables occupants within offices to notify the BMS or a building manager about the state of their thermal comfort and consequentially suggest necessary actions. Furthermore, motivated by the same paradigm and to support the differential pricing due to renewable energy supply, Alan et al. [32] conducted a field study with three smart thermostat prototypes in 30 UK residences to comprehend the mental models and expectations about inhabitants' thermal comfort. Costanza et al. with their Temperature Calendar [33] - an ambient visualization of thermal variations in workplaces - have used these displays as a medium for occupants to engage with the policy makers, and collectively appropriate workplaces that offer higher thermal comfort while optimizing energy resources.

Respiratory comfort corresponding to the surrounding air quality, has been examined with (quite some) urgency in the last several years, primarily to mitigate the adverse effects of air pollution in urban environments, and to support occupants' "right to awareness" about the healthiness of their surroundings. Within buildings, awareness systems that register and visualize the levels of Particulate Matter (for example, inAir [34], [35]) and Carbon Dioxide - CO2 - (for example, MAQS [36]) have been designed and evaluated. In addition to monitoring, the weather station developed by Frešer et al. [37] also recommends the relevant future actions to ensure a healthy environment by additionally accounting for the number of occupants within a room and the current state of windows.

\footnotetext{
${ }^{8}$ Heating, Ventilation, and Air-Conditioning
} 
The main track of comfort research in architecture differs markedly from the perspective of HCI and its emphasis on the active role of occupants in achieving their comfort. The schema that we demonstrate in the next section provides a bigger picture that incorporates components relevant to both perspectives while articulating the distinctions.

\section{Occupant-Centered Model of Comfort}

In the model that we illustrate in Figure 1, the individual occupant (user) seeks comfort in continuous interaction with her environment. The environment consists of the physical situation, but also the social setting, that is, the other occupants are considered as part of the environment. The model can be best understood by tracing two processes of comfort evaluation, which we discuss in the following as Involuntary and Voluntary:

1. Involuntary Evaluation - Autonomic Response A continuous sensing process monitors the part of the physical environment inside occupant's receptive field (e.g. intensity of light in her field of view, temperature in her micro-environment). This is done by the human's sensory system, which consists of several components: the sensory endings (e.g. photoreceptors in retina, thermoreceptors in skin), neural pathways, part of the brain that perceives the transduced sensory information, and a transient sensory memory (e.g. iconic memory, haptic memory) [38]. Depending on the external situation perceived in the environment and the internal state of the occupant (physiological and psychological), comfort at that moment is evaluated involuntarily. Consequently a set of regulatory actions take place, in order to maintain a relatively constant condition for the inner organs. These actions are autonomic and may be in the form of $a$ ) physiological responses (e.g. retina size change, squinting, sweating, shivering, heart rate change), and/or $b$ ) impact on the psychological state (e.g. annoyance), which may trigger a "waking signal", calling for (sub)conscious evaluation of situation and behavioral response.

2. Voluntary Evaluation - Behavioral Response There are occasions when comfort fades into our conscious or subconscious attention. This may stem from the fact that involuntary responses have not been sufficient (as mentioned above), but also due to external triggers such as being the subject of a comfort study and asked to rate it, or even at a meta-cognition level, for example, when reading this text. Several parameters may impact our voluntary judgment of comfort: $a$ ) the physiological and psychological state (e.g. sweating, being annoyed by noise), $b$ ) sensory information, and also $c$ ) the mental model of the environment related to the level of expectations and tolerance. The outcome may appear as interaction with the built environment (e.g. opening a window, closing the shutter).

These actions are intended to make modification in body's immediate environment and may update the occupant's mental model of her environment and how it reacts to various manipulations.

Without aspiring to delineate these mechanisms (some of which are still open scientific questions), we argue that by recognizing the distinction between the Involuntary and

Voluntary states, the model highlights points that are of special interest to the discussion of comfort in the context of Human-Building Interaction. 


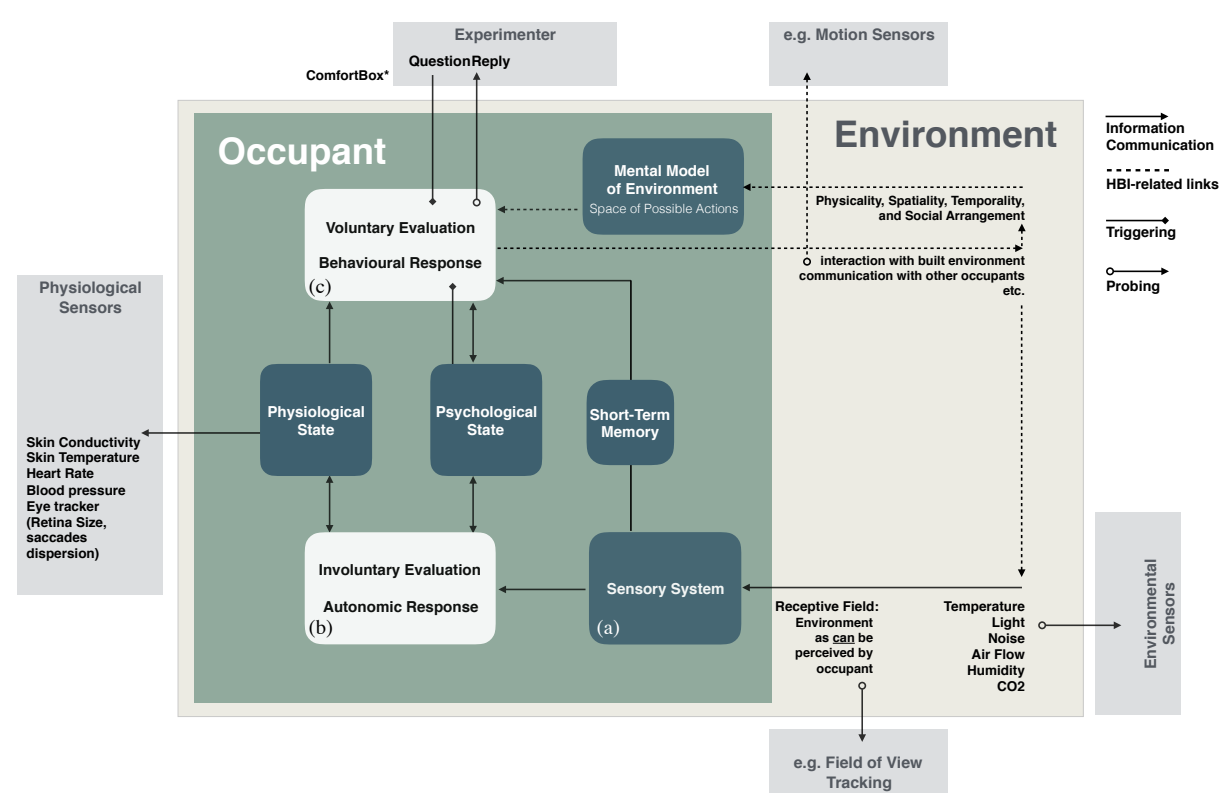

Fig. 1. Comfort is achieved in interaction with the built environment, through an iterative process in which the occupants (a) observe the environmental condition with their sensory receptors, (b) evaluate it involuntary, and accordingly change physiological and/or psychological state, and if remarkable discomfort is observed, (c) (sub)consciously acts upon it, given the space of possibilities that the occupants believe they have. The adaptation action may make a change in the environment objectively and in the occupants' perception. The "Voluntary Evaluation" state may also be triggered by an external factor such an experimenter asking the occupants to reflect about their comfort.

In addressing an HBI design question such as "what style of interaction with a window-like element can enhance the occupant's comfort?" what is at stake is the moments when a transition from Involuntary to Voluntary occurs, leading to a behavioral response that may be in the form of interacting with the designed element. While a performant building should minimize the instances when comfort becomes a (sub)conscious concern, the role of interaction design is to serve those moments, to understand the occupant's physical and mental state in those situations, to create appropriate space of possible actions, to offer "usable" interactivity, and to help the concern of comfort fade back to the Involuntary state.

HBI studies must be able to capture (or deliberately generate) the situations where comfort is in the Voluntary state. They must also be able to understand the possible correlations between the alternative design choices and the user behaviour in those situations. However, for the sake of (holistic) completeness, it is critically (and yet 
equally) important for an HBI researcher to create a full understanding of episodes that lead to the transitions to and from the Involuntary state. Consequently, a contextualized, sensitized, and localized understanding of each individuals' surroundings and physiological measurements, might allow for inference of ones' level of comfort - information that can be queried in the findings reviewed in Section 2 (especially the answers to the broad questions $Q 1$ and $Q 4$ ). We continue discussing this model in the next section, by explaining two methodological concerns that it uncovered, motivating the design of ComfortBox.

\section{ComfortBox as a Research Tool}

Motivation. The model pronounces two methodological concerns in conducting comfort surveys, when asking the subject of the study, for instance, "how do you rate your thermal comfort?" First, our sensory memory is highly transient, and the participants' answer to that question would reflect only their experience in the last moments before the question was asked. Given that, the answer to a question such as "how do you rate visual comfort during the last week?" is highly unreliable. This makes aligning the questionnaire data with the observed user's behaviour to be practically impossible, especially in longitudinal studies. On the other hand, frequent prompting could not be an appropriate solution, as it creates annoyance and itself affects comfort and the way it is reported. Second, when the experimenter asks the study subjects to rate their comfort, this prompting triggers the Voluntary process of comfort evaluation. Activating the Voluntary process is part of the biological mechanism to call for behavioral response. An external (artificial) trigger might raise concerns about the ecological-validity of the study, and might simply result in exaggerated responses.

Design Concept. As opposed to questionnaires that are collected at fixed points in time during the course of the study (typically at the start and/or the end), ComfortBox allows the experimenters to ask questions at particular "(intelligently) identified moments". For example, the experimenter can decide that a certain question should be asked when the subject opens a window or changes the thermostat setting. From observing such behavioral responses, one can speculate that the participant is already in the Voluntary state, (avoiding the first concern: ecological-validity), and also can collect data that corresponds to the time when a relevant action is monitored (addressing the second problem: data synchronization). The question that appears on the screen can be designed by the experimenter for that situation and the answers are given directly using the feedback buttons on top of the device.

Technical Specification. ComfortBox, shown in Figure 2, is a 10x10x10cm cube (3 sides wooden and 3 sides mirror surfaces). It contains a set of environmental sensors (temperature, humidity, airflow, air pressure, light, $\mathrm{CO} 2$, and noise) that send measurements every second to a cloud-based database, via WiFi and MQTT 99 protocol. An OLED screen on the front side and a ring of LEDs can display text or color-coded information. The displayed information (question) is fed to the ComfortBox by an algorithm that

\footnotetext{
${ }^{9}$ http://mqtt.org/
} 

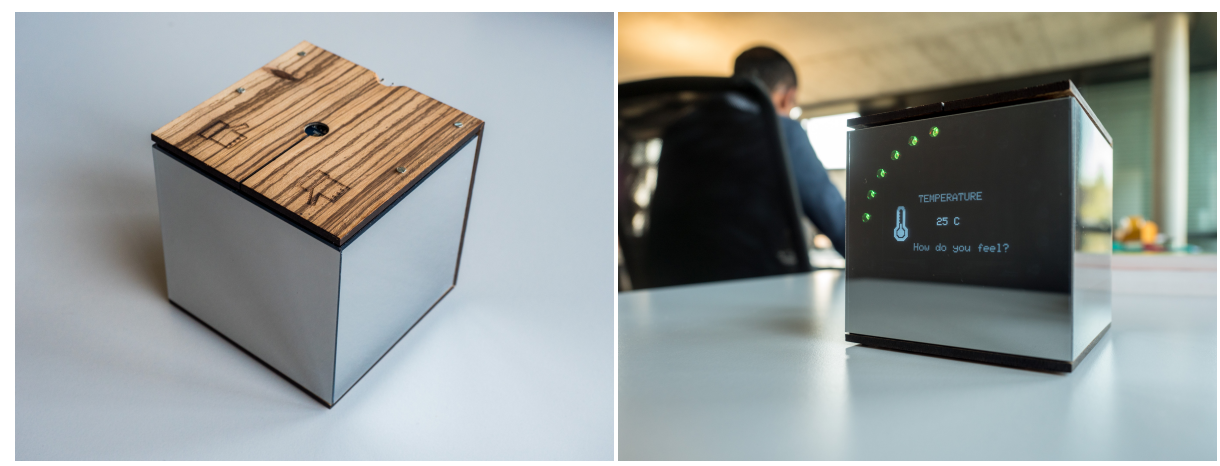

Fig. 2. ComfortBox is an interactive research tool designed to facilitate data collection in lab and in-situ comfort studies. The physical design is intended to help blend into the desktop setting and stay in the periphery of attention when not used.

runs on a MQTT server. This algorithm is tinkered by the experimenter and can work with input variables from the ComfortBoxes' sensor measurements, but also from other sensors used in the study. The buttons on top of the box allow for answering the posed questions.

API for Researchers. A programming interface based on Node-RED ${ }^{10}$ is provided. The experimenter designs a user scenario which determines the opportune circumstances under which a specific question can be asked, and particular information to be provided to the user.ComfortBox is currently being used by the Human-IST research center, in University of Fribourg, Switzerland, in two ongoing research projects that aim to address the impact of "agency" and "awarness" on the perception of comfort. ComfortBox contributes to the model (illustrated in Figure 1) at the same position as the "Experimenter" component. It substitutes (or supports) the survey mechanism, with the possibility to connect to the other sensing systems (e.g. physiological, environmental, and motion sensors).

\section{Conclusion}

At the core of this contribution, our proposed model extends the generally accepted view in architectural studies which considers comfort as a "quality of building", and suggests that comfort is also an "objective for occupants", to be achieved in interaction with the built environment. It illustrates that besides the involuntary physiological and psychological processes, an independent yet communicating conscious process contributes to the accomplishment of comfort. And finally, of high relevance to HumanBuilding Interaction discourses, the model recognizes that comfort has autonomic but also behavioral manifestations; that it is part of our interactive experience with the built environment and entails design attempts that account for its full picture.

\footnotetext{
${ }^{10}$ https://nodered.org/
} 


\section{Bibliography}

[1] Paul Dourish. Where the action is: the foundations of embodied interaction. MIT press, 2004.

[2] Brett Ingram. Feature-learning from architecture. interactions, 16(6):64-67, 2009.

[3] Christopher Alexander, Sara Ishikawa, Murray Silverstein, Joaquim Romaguera i Ramió, Max Jacobson, and Ingrid Fiksdahl-King. A pattern language. Gustavo Gili, 1977.

[4] Stewart Brand. How buildings learn: What happens after they're built. Penguin, 1995.

[5] William J Mitchell. City of bits: space, place, and the infobahn. MIT press, 1996.

[6] Neil Spiller et al. Digital architecture now: A global survey of emerging talent. Thames \& Hudson, 2008.

[7] Hamed S Alavi, Elizabeth Churchill, David Kirk, Julien Nembrini, and Denis Lalanne. Deconstructing human-building interaction. Interactions, 23(6), 2016.

[8] Himanshu Verma, Hamed Alavi S., and Denis Lalanne. Studying Space Use: Bringing HCI Tools to Architectural Projects. In Proc. of CHI'17. ACM, 2017.

[9] Hamed Alavi, Denis Lalanne, Julien Nembrini, Elizabeth Churchill, David Kirk, and Wendy Moncur. Future of human-building interaction. In Proc. of CHI'16 Extended Abstracts. ACM, 2016.

[10] Dean Hawkes. The environmental imagination: technics and poetics of the architectural environment. Taylor \& Francis, 2008.

[11] Philomena M Bluyssen. The indoor environment handbook: how to make buildings healthy and comfortable. Earthscan, 2009.

[12] P.O. Fanger. Thermal Comfort. McGraw-Hill, New York, danish tec edition, 1970.

[13] Ralph Galbraith Hopkinson. Architectural physics: Lighting. 1963.

[14] Carl-Gustaf Bornehag, J Sundell, C Weschler, T Sigsgaard, B Lundgren, M Hasselgren, and L Hagerhed-Engman. The association between asthma and allergic symptoms in children and phthalates in house dust: A nested case-control study. Environmental Health Perspectives, 112(14):1393-1397, 2004.

[15] W. J. Fisk, Q. Lei-Gomez, and M. J. Mendell. Meta-analyses of the associations of respiratory health effects with dampness and mold in homes. Indoor Air, (4), 2007.

[16] Richard J De Dear, Gail Schiller Brager, James Reardon, Fergus Nicol, et al. Developing an adaptive model of thermal comfort and preference/discussion. ASHRAE transactions, 104:145, 1998.

[17] ASHRAE Standard. Standard 55-2013. Thermal environmental conditions for human occupancy, 2013.

[18] CEN. Indoor environmental input parameters for design and assessment of energy performance of buildings addressing indoor air quality, thermal environment, lighting and acoustics. European Committee for Standardization, Belgium, 2007.

[19] CEN. Light and lighting-lighting of work places-part 1: Indoor work places. European Committee for Standardization, Brussels, Belgium, 2002.

[20] EN CEN. Acoustics - description, measurement and assessment of environmental noise - part 1: Basic quantities and assessment procedures. European Committee for Standardization, Brussels, Belgium, 2016. 
[21] John E Janssen. Ventilation for acceptable indoor air quality. ASHRAE journal, 31(10):40-48, 1989.

[22] Raymond. Cole and Richard. Lorch. Buildings, Culture and Environment. John Wiley \& Sons, 2007.

[23] Heather Chappells and Elizabeth Shove. Debating the future of comfort: environmental sustainability, energy consumption and the indoor environment. Building Research \& Information, 33(1):32-40, 2005.

[24] Arezou Shafaghat, Ali Keyvanfar, Mohamed Salim Ferwati, and Tooran Alizadeh. Enhancing staff's satisfaction with comfort toward productivity by sustainable Open Plan Office Design. Sustainable Cities and Society, 19:151-164, aug 2015.

[25] Wolfgang FE Preiser, Edward White, and Harvey Rabinowitz. Post-Occupancy Evaluation (Routledge Revivals). Routledge, 2015.

[26] A Wagner, E Gossauer, C Moosmann, Th Gropp, and R Leonhart. Thermal comfort and workplace occupant satisfaction results of field studies in german low energy office buildings. Energy and Buildings, 39(7):758-769, 2007.

[27] P Bluyssen, M Aries, and P Dommelen. Comfort of workers in office buildings: The European HOPE project. Building and Environment, 46(1):280-288, 2011.

[28] Ye Yao, Zhiwei Lian, Weiwei Liu, and Qi Shen. Experimental study on physiological responses and thermal comfort under various ambient temperatures. Physiology \& Behavior, 93(1):310-321, 2008.

[29] Y Yao, Z Lian, W Liu, C Jiang, Y Liu, and H Lu. Heart rate variation and electroencephalograph-the potential physiological factors for thermal comfort study. Indoor air, 19(2):93-101, 2009.

[30] Carl DiSalvo, Phoebe Sengers, and Hrönn Brynjarsdóttir. Mapping the landscape of sustainable HCI. In Proc. of CHI'10, pages 1975-1984. ACM, 2010.

[31] Milan Milenkovic, Ulf Hanebutte, Yonghong Huang, David Prendergast, and Han Pham. Improving user comfort and office energy efficiency with POEM (Personal Office Energy Monitor). In CHI'13 Extended Abstracts. ACM, 2013.

[32] Alper T. Alan, Mike Shann, Enrico Costanza, Sarvapali D. Ramchurn, and Sven Seuken. It is too hot: An in-situ study of three designs for heating. In Proc. of CHI'16, pages 5262-5273. ACM, 2016.

[33] Enrico Costanza, Ben Bedwell, Michael O. Jewell, James Colley, and Tom Rodden. 'a bit like british weather, i suppose': Design and evaluation of the temperature calendar. In Proc. of CHI'16, pages 4061-4072. ACM, 2016.

[34] Sunyoung Kim and Eric Paulos. inAir: Measuring and visualizing indoor air quality. In Proc. of UbiComp'09, pages 81-84. ACM, 2009.

[35] Xuxu Chen, Yu Zheng, Yubiao Chen, Qiwei Jin, Weiwei Sun, Eric Chang, and Wei-Ying Ma. Indoor air quality monitoring system for smart buildings. In Proc. of UbiComp'14, pages 471-475. ACM, 2014.

[36] Yifei Jiang, Kun Li, Lei Tian, Ricardo Piedrahita, Xiang Yun, Omkar Mansata, Qin Lv, Robert P. Dick, Michael Hannigan, and Li Shang. MAQS: A mobile sensing system for indoor air quality. In Proc. of UbiComp'11. ACM, 2011.

[37] Martin Frešer, Anton Gradišek, Božidara Cvetković, and Mitja Luštrek. An intelligent system to improve T-H-C parameters at the workplace. In Proc. of UbiComp'16 Adjunct, pages 61-64. ACM, 2016.

[38] John Krantz. Experiencing sensation and perception. Pearson Education (Us), 2012. 